\title{
Synthesis and Characterization of Polyhedral Graphite Particles
}

\author{
Wen Qian, Qianwang Chen *, Fangyu Cao and Changle Chen \\ Hefei National Laboratory for Physical Sciences at Microscale and Department of Materials Science \& Engineering, \\ University of Science \& Technology of China, Hefei 230026, P.R. China
}

\begin{abstract}
Hollow polyhedral graphite particles with $200 \sim 400 \mathrm{~nm}$ in diameter have been synthesized by heating oxalic acid, ferrocene and metallic magnesium at $580{ }^{\circ} \mathrm{C}$, typically forming diamond-like octahedral structure. Combined with SEM, TEM, SAED patterns, and Raman spectroscopy, it was shown that the surface of graphite polyhedrons is composed of approximately 30-40 graphene shells; the four- or six- fold symmetric diffraction spots disclosed the nature of octahedral graphite particles; the edge of the graphite polyhedrons exists $\mathrm{sp}^{3}$-hybridized bonding, which would act as dangling bonds and localized defects in the polyhedron.
\end{abstract}

Keywords: Nanostructures, chemical synthesis, crystal structure and symmetry.

\section{INTRODUCTION}

While the great stability of carbon-carbon bonds has been recognized for more than 100 years, during very long time after this recognition, polyhedral diamond and lamellar graphite were the only allotropic forms of carbon. In diamond, a typical polyhedral carbon elemental crystal, the four bonds to each carbon atom are arranged in an extremely strong three-dimensional (3-D) matrix. Due to the 3-D structure and the symmetrical arrangement of atoms, diamond crystals usually develop into several different polyhedral shapes including octahedron, cubes, dodecahedra, and combinations of them, known as 'crystal habits'. In contrast, the feature is quite different for the 2-D lamellar graphite, which consists of individual graphene layers. Within a single graphene layer, each carbon atom is tightly bonded to three atoms forming a 2-D flat hexagonal network, while between the graphene layers there is weakly van der Waals force, therefore neighboring graphene layers can slide easily. The synthesis of novel allotropic carbon forms has long been of considerable interest. Synthesis of bulk quantities of $s p^{2}$ bonded 0-D fullerene molecules [1] and 1-D nanotubes [2] has demonstrated that abundant structures existing in carbon family, and new forms of carbon is possible [3, 4]. Because of the great difference between the force of $\mathrm{sp}^{2} \mathrm{C}-\mathrm{C}$ bond in layer and the van der Waals force between layers, it is hard for graphite-like $\mathrm{sp}^{2}$ carbon atoms to form a stable diamondlike 3-D feature. However, theoretical calculations have suggested the possibility of fairly stable covalent $\mathrm{sp}^{2}$ carbon crystals such as schwarzites [5] and hollow graphites [6] into a fully $3 \mathrm{D}$ architecture, which have given new perspectives on the structure of $\mathrm{sp}^{2}$ graphitic carbon [7]. Recently, several polyhedral graphite consisting of discrete fragments of curved graphitic sheets have been obtained, such as graphite polyhedral tubular crystals (GPC) with $1 \sim 3 \mu \mathrm{m}$ in diameter and $5 \sim 15 \mathrm{~mm}$ in length $[8,9]$ and polyhedral graphite particles (called ' $G$ balls') with $300 \mathrm{~nm}$ in diameter [10].

*Address correspondence to this author at the Hefei National Laboratory for Physical Sciences at Microscale and Department of Materials Science \& Engineering, University of Science \& Technology of China, Hefei 230026, P.R. China; E-mail: cqw@ustc.edu.cn
Herein, we report a chemically synthesized novelty of graphitic nanoparticles with diamond-like polyhedral structure, typically forming octahedron. The polyhedral graphite particles is as large as 200 400 nm, exhibiting a 3-D symmetrical feature quite different from a 2-D crystal. The unique microstructure characteristics about the polyhedral graphite particles were also discussed.

\section{EXPERIMENTAL SECTION}

Ferrocene (98\%), oxalic acid (99.95\%), ethanol, hydrochloric acid (analytically pure) and metallic magnesium ( $98 \%$, chemically pure) were all used as purchased. The typical reaction was carried out by heating a mixture of 3.00 $\mathrm{g} \mathrm{H}_{2} \mathrm{C}_{2} \mathrm{O}_{4}, 1.50 \mathrm{~g} \mathrm{Fe}\left(\mathrm{C}_{5} \mathrm{H}_{5}\right)_{2}$ and $0.80 \mathrm{~g}$ metallic $\mathrm{Mg}$ in a stainless-steel autoclave with the capacity of $15 \mathrm{ml}$ at $580{ }^{\circ} \mathrm{C}$ for $10 \mathrm{~h}$, and then cooling down to room temperature naturally. The as-prepared products were gray in color; then washed with ethanol, distilled water and $4.0 \mathrm{~mol} / \mathrm{L} \mathrm{HCl}$ aqueous solution respectively; afterwards the precipitate was collected by using a microfilter; followed by washed with distilled water for several times, until the $\mathrm{pH}$ value was about 6 ; finally dried at $110^{\circ} \mathrm{C}$.

The morphology and structure of polyhedral graphitic particles were observed by Field emission scanning electron microscope (FESEM) using a JEOL JSM-6300F SEM, a Hitachi Model H-800 Transmission electron microscope (TEM) with Electron Diffraction, and a JEOL-2010 highresolution TEM with Energy Dispersive X-ray Detector, using an accelerating voltage of $200 \mathrm{kV}$. The Raman spectrum was taken on a LABRAM-HR Confocal Laser MicroRaman spectrometer using an $\mathrm{Ar}^{+}$laser with $514.5 \mathrm{~nm}$ at room temperature.

\section{RESULTS AND DISCUSSION}

Shown in the XRD pattern (Fig. 1), it is obvious that after the treatment with $\mathrm{HCl}$ aqueous solution, the main product of the sample is hexagonal graphite. Fig. (2a) shows TEM images of typical polyhedral particles with the average size of $300 \mathrm{~nm}$ after $\mathrm{HCl}$ treatment (Fig. 2a) and as-prepared products (Fig. 2a, insert) respectively. Fig. (2b) shows FESEM images of various graphite polyhedron, in which hexahe- 
dron, octahedron, and dodecahedron can be clearly seen. According to TEM and FESEM images observed, the fraction of octahedral particles among the polyhedral particles was approximately 20\%. Corresponding EDX spectrum (Fig. 3) shows $\mathrm{C}$ and $\mathrm{Cu}$ peaks only, in which $\mathrm{Cu}$ signal originated from carbon-coated copper grids. Associated with XRD pattern, the EDX spectrum analysis can confirm that the polyhedral particles are graphite. Fig. (2c) displays the TEM images of typical graphite polyhedron, and (Fig. 2d) is the magnified HRTEM image of boxed area of (Fig. 2c). The thickness of each face of the octahedron is about 30-40 atomic layers. The inter-sheet between the layers is about $0.35 \mathrm{~nm}$, agrees well with (002) lattice distance in hexagonal graphite $(0.34 \mathrm{~nm})$. As to the joint edge between adjacent faces, there exists a discontinuous crystal boundary, which is different from the lattice structure of adjacent faces. Similar results were also found in repeated experiments.

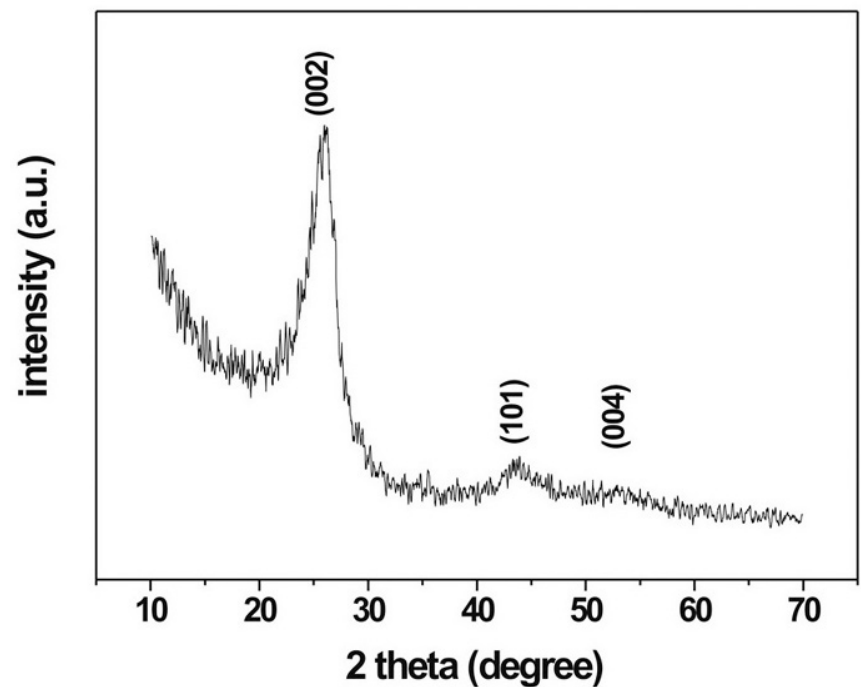

Fig. (1). The XRD pattern of the sample, recorded in the $2 \theta$ range of $10-70^{\circ}$ by using a Rigaku (Japan) D/max- $\gamma$ A X-ray diffractometer equipped with graphite monochromatized $\mathrm{Cu} \mathrm{K}$-alpha radiation $(\lambda=1.542 \AA)$.

It is worthy to mention the SAED patterns. Though there is six-fold symmetry in a graphene sheet, the six-fold symmetry is rarely found in the diffraction patterns of graphite because the slide between graphene sheets in graphite destroys the sixfold symmetry in it. Practically, no diffraction spot exists when incident electronic beam is along the (002) orientation of a graphitic single crystal. When incident electronic beam is vertical to the (002) orientation, the diffraction of traditional single crystalline graphite usually shows bisymmetric spots, while polycrystalline graphite shows a diffraction ring. When it comes to carbon nanotubes, the electronic diffraction spots disperse to two small symmetric arcs, whose length depends on the stress of the warped graphene layers. More than two diffraction spots of graphite (002) in one pattern are rarely observed. However, SAED patterns of the octahedral graphitic particles (inset of Fig. 4), show four- or six- fold symmetric spots corresponding to hexagonal graphite $(002)(\mathrm{d}=3.5 \AA)$, quite different from traditional graphite particles. Since there is no axis of tetragonal symmetry in hexagonal graphitic crystal, these diffraction spots should be resulted from the symmetry of the octahedral particles. Shortly speaking, the emergence of the fourfold or higher symmetric short diffraction arcs is absolutely the unique character of polyhedral graphite particles.

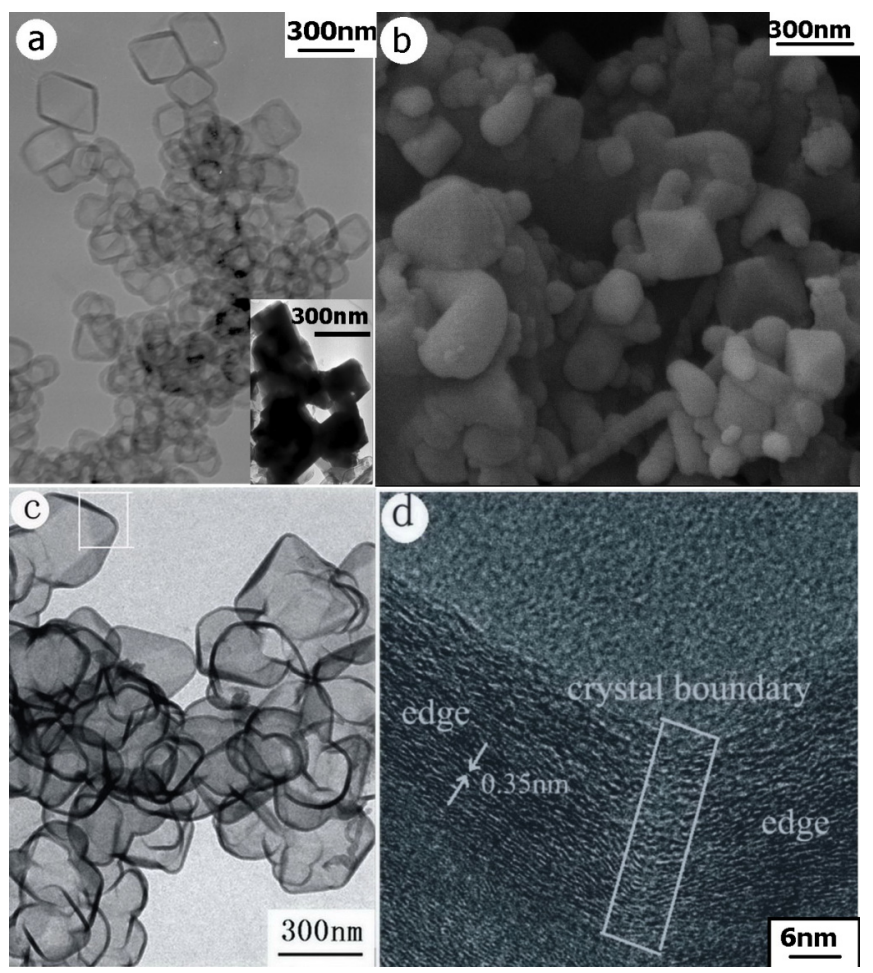

Fig. (2). (a,b) TEM and FESEM images of large quantities of polyhedral graphite particles with the average size of $300 \mathrm{~nm}$; (c) TEM images of typical graphite polyhedron; (d) The magnified HRTEM image of boxed area shown in (c), shows the discontinuous crystal boundary of polyhedral graphite particle.

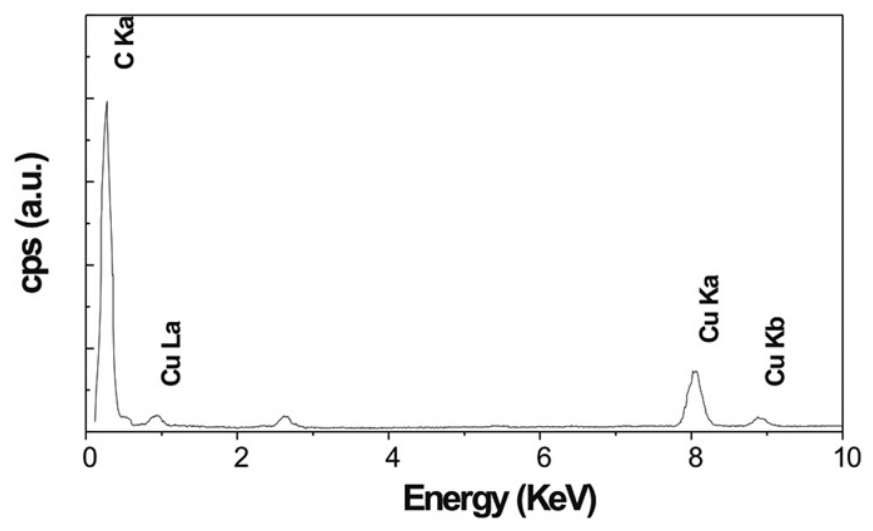

Fig. (3). The typical EDX spectrum of a polyhedral graphite particle, analyzed by a JEOL-2010 HRTEM with Energy Dispersive Xray Detector.

Raman spectrum is usually used to investigate the vibrational properties of carbon structures, which also allows us to draw further conclusion about the degree of crystallization [11]. Fig. (5) represents the experimental Raman spectrum of the sample, as well as the fitting results which were obtained by using the Gaussian method. It is found that the main spectrum can be decomposed into three sets, with the peaks of 


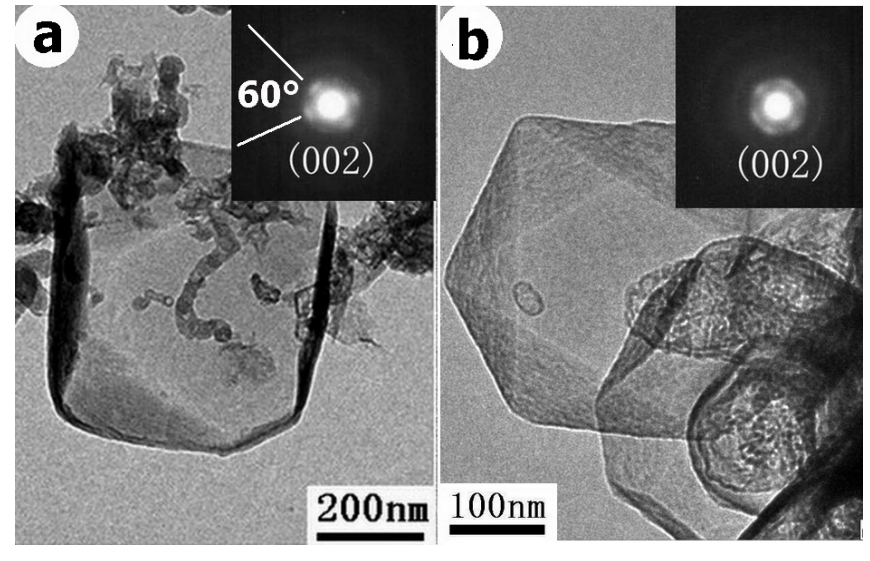

Fig. (4). (a,b) The typical octahedral-like and polyhedral graphite particles, and the corresponding SAED pattern (insert) consist of four and six diffraction short arcs in the presence of weak diffuse rings respectively.

1332,1350 , and $1598 \mathrm{~cm}^{-1}$. The peak at $\sim 1598 \mathrm{~cm}^{-1}$ (G band) corresponding to an $\mathrm{E}_{2 \mathrm{~g}}$ mode of graphite is related to the vibration of $\mathrm{sp}^{2}$-bonded carbon atoms in a 2-D hexagonal lattice, while the peak at $\sim 1350 \mathrm{~cm}^{-1}$ (D band) is related to the defects and disorders in the hexagonal graphitic layers [12]. In Fig. (5), the value of $\mathrm{I}_{\mathrm{D}} / \mathrm{I}_{\mathrm{G}}$ is calculated to be 0.63 , which means that the sample has the relative disorder and not very highly graphitization degree [13], in agreement with $\mathrm{XRD}$ results. Furthermore, it is worthy to be noticed that the peak at $\sim 1332 \mathrm{~cm}^{-1}$ corresponds to $\mathrm{sp}^{3}$-hybridized bonds, indicating the existence of $\mathrm{sp}^{3}$ bonds environment. Based on theoretical calculations simulating the closed graphitic structures, both De Vita et al and V. L. Kuznetsov et al had put forward that the $\mathrm{sp}^{3}$ and $\mathrm{sp}^{2}$ bonds can coexist, and $\mathrm{sp}^{3}$ bonds are located at peripheries of graphitic $\mathrm{sp}^{2}$ sheets $[14,15]$. G. Benedek et al also predicted the possible $s p^{2} / s p^{3}$ mixed bonding existing in hollow carbon crystals [6]. All of the above theoretical computations disclosed that the joint edge between adjacent faces of polyhedral graphite particle is likely not composed of $\mathrm{sp}^{2}$ bond, but connected by $\mathrm{sp}^{3}$ hybridized bonds, which would act as dangling bonds and localized defects in the polyhedron [16].

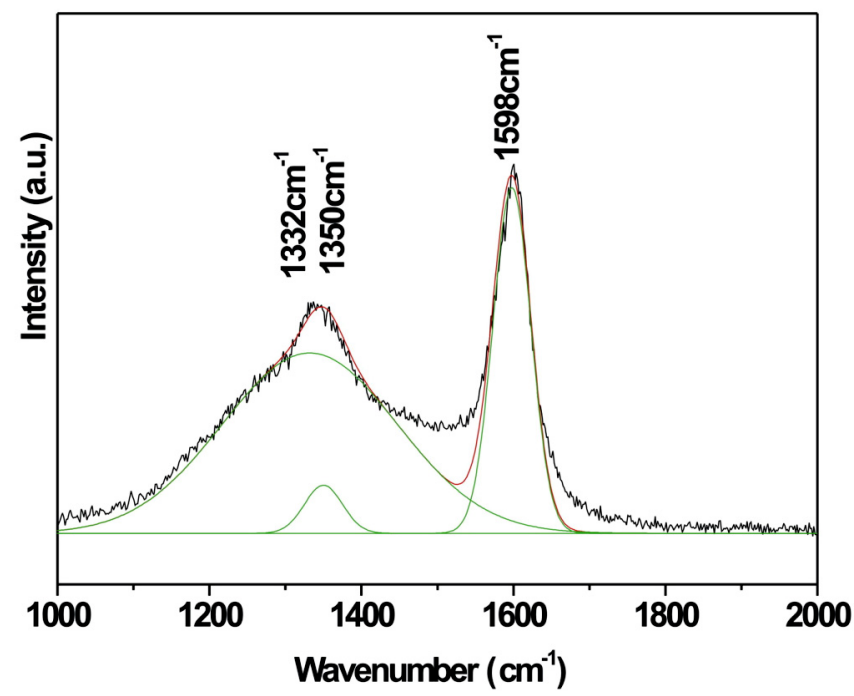

Fig. (5). Typical Raman spectrum and corresponding fitting results of the sample.
In addition, TEM images and SAED patterns have revealed that the polyhedral graphite particles are hollow inside, as shown in Scheme 1. Each face of the hollow polyhedron consists of graphene multilayers (approximately 30-40 shells). When the incident electron beam is along the edge of one polyhedron, paralleling to the two faces sharing the edge, SAED pattern shows four graphite (002) diffraction spots, which are two series of bisymmetric spots with the center spot as the symmetric center. The separation angle of two corresponding spots from the center spot, which is $60^{\circ}$ (Fig. 4a), is equal to the dihedral angle formed by two adjacent faces of a polyhedron, showing that the polyhedron is octahedron. When a facet of another octahedron is near and parallel to the electronic beam, two more bisymmetric spots may present in the diffraction pattern, as shown in Fig. (4b).
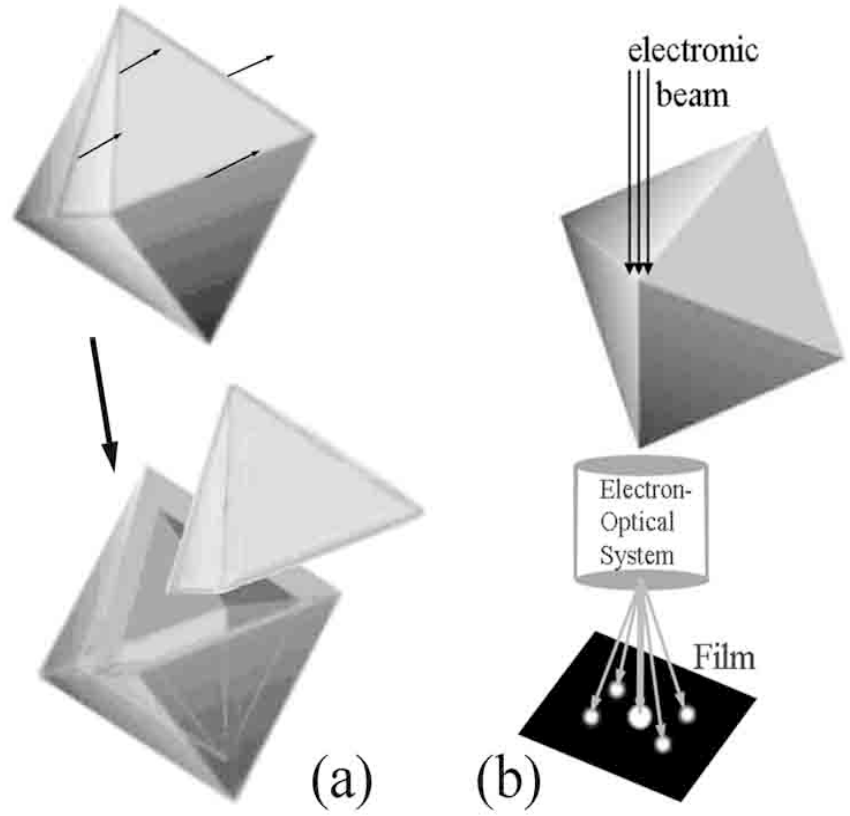

Scheme 1. (a) Structural model of hollow octahedral graphite particle, consisting of eight plate-like graphite single crystals; and (b) Model of the appearance of the four-fold electronic diffraction spots.

These polyhedral graphite particles cannot be obtained without the present of $\mathrm{H}_{2} \mathrm{C}_{2} \mathrm{O}_{4}$ or ferrocene, indicating that carbon in $\mathrm{H}_{2} \mathrm{C}_{2} \mathrm{O}_{4}$ and ferrocene plays different role in the growth of the polyhedrons. As to the possible growth model of polyhedral graphite particles, we would suggest as following: first, $\mathrm{H}_{2} \mathrm{C}_{2} \mathrm{O}_{4}$ is easily decomposed to $\mathrm{CO}_{2}, \mathrm{CO}$, and $\mathrm{H}_{2} \mathrm{O}$ when heated over $100{ }^{\circ} \mathrm{C}$, and $\mathrm{CO}$ and $\mathrm{CO}_{2}$ were deoxidized to carbon by metallic Mg slowly, forming initial closed nucleus due to pentagons and heptagons giving rise to a curved geometry at the edges [17]; then catalyzed by iron atoms or iron carbide originating from ferrocene [18], more and more carbon atoms were deposited simultaneously at high temperature, leading to possible concentric growth; eventually terminating with the formation of $\mathrm{Fe}$ or $\mathrm{Fe}_{3} \mathrm{C}$ nano-crystals encapsulated in polyhedral carbon cages [19,20], as shown in Fig. (2a) insert. Finally, the as prepared products were treated with $\mathrm{HCl}$ aqueous solution, so $\mathrm{Fe}$ or $\mathrm{Fe}_{3} \mathrm{C}$ nanocrystals were removed, forming hollow graphite polyhedron. Further work is still needed to figure out the growth mechanism and illustrate the structure of polyhedral graphite particle clearly. 


\section{CONCLUSIONS}

In conclusion, we have successfully synthesized the novel hollow graphite particles with polyhedral feature by introducing oxalic acid, ferrocene and metallic magnesium at $580{ }^{\circ} \mathrm{C}$, especially some of them were octahedron-like in shape. The surface of the hollow graphite octahedron is composed of concentric $\mathrm{sp}^{2}$ graphitic shells, but at the crystal boundary, there exists $\mathrm{sp}^{3}$-hybridized bonds, which would act as dangling bonds and localized defects. The easily synthesized octahedral graphite particles reported in this letter would give us a new understanding of the 'crystal habits' that a two dimensional crystal like graphite may also crystallize in form of diamond-like three dimensional feature. Moreover, the octahedral graphite would be added in to the family of elemental carbon as a new member, like fullerenes and carbon nanotubes.

\section{ACKNOWLEDGEMENT}

This work was supported by the National Natural Science Foundation of China.

\section{REFERENCES}

[1] Kroto HW, Heath JR, O'Brien SC, Curl RF, Smalley RE. C60: Buckminsterfullerene. Nature 1985; 318(14): 162-163.

[2] Iijima S. Helical microtubules of graphitic carbon Nature 1991; 354(6348): 56-58

[3] O'Keeffe M, Adams GB, Sankey OF. Predicted new low energy forms of carbon. Phys Rev Lett 1992; 68(15): 2325-2328.

[4] Fayos J. Possible 3D carbon structures as progressive intermediates in graphite to diamond phase transition. J Solid State Chem 1999; 148(2): 278-285.

[5] Vanderbilt D, Tersoff J. Negative-curvature fullerene analog of $\mathrm{C}_{60}$. Phys Rev Lett 1992; 68(4): 511-513.
Benedek G, Colombo L, Gaito S, Galvani E, Serra S. Prediction of new sp2 and $\mathrm{sp}^{2} / \mathrm{sp}^{3}$ hollow carbon crystals. J Chem Phys 1997; 106(6): 2311-2316.

[7] Harris PJF. New perspectives on the structure of graphitic carbons. Crit Rev Solid State Mater Sci 2005; 30(4): 235-253.

[8] Gogotsi Y, Libera JA, Kalashnikov N, Yoshimura M. Graphite polyhedral crystals. Science 2000; 290(5490): 317-320.

[9] Okuno H, Palnichenko A, Despres JF, Issi JP, Charlier JC. Synthesis of graphite polyhedral crystals using a combustion flame method. Carbon 2005; 43(4): 692-697.

[10] Kokai F, Takahashi K, Kasuya D, et al. Laser vaporization synthesis of polyhedral graphite. Appl Phys A: Mater Sci Process 2003; 77(1): 69-71.

[11] Andersson OE, Prasad BLV, Sato H, et al. Structure and electronic properties of graphite nanoparticles. Phys Rev B 1998; 58(24): 16387-16395.

[12] Klinke C, Kurt RJ, Bonard M, Kern K. Raman spectroscopy and field emission measurements on catalytically grown carbon nanotubes. J Phys Chem B 2002; 106(43): 11191-11195.

[14] De Vita A, Galli G, Canning A, Car R. A microscopic model for surface-induced diamond-to-graphite transitions. Nature 1996; 379(6565): 523-526.

[15] Kuznetsov VL, Zilberberg IL, Butenko YV, Chuvilin AL, Segall B Theoretical study of the formation of closed curved graphite-like structures during annealing of diamond surface. J Appl Phys 1999; 86(2): 863-870.

[16] Tomita S, Sakurai T, Ohta H, Fujii M, Hayashi S. Structure and electronic properties of carbon onions. J Chem Phys 2001; 114 (17) 7477-7482.

[17] Xiong YJ, Xie Y, Li XX, Li ZQ. Production of novel amorphous carbon nanostructures from ferrocene in low-temperature solution. Carbon 2004; 42(8-9): 1447-53.

[18] Hou H, Schaper AK, Weller F, Greiner A. Carbon nanotubes and spheres produced by modified ferrocene pyrolysis. Chem Mater 2002; 14(9): 3990-4.

[19] Seraphin S, Zhou D, Jiao J. Filling the carbon nanocages. J Appl Phys 1996; 80(4): 2097-2104.

[20] Geng JF, Jefferson DA, Johnson BFG. Direct conversion of iron stearate into magnetic $\mathrm{Fe}$ and $\mathrm{Fe} 3 \mathrm{C}$ nanocrystals encapsulated in polyhedral graphite cages. Chem Commun 2004; 10(21): 24422443. 